\title{
Solvent Free Quick Conversion of Mono - And Dicarboxylic Acids into Their Corresponding Anilides with Phenylisothiocyanate
}

\author{
Gunanka Hazarika, Pradeep K. Tripathy* \\ Department of Chemistry, North Eastern Regional Institute of Science and Technology, Nirjuli, Itanagar (Arunachal Pradesh), India \\ Email address: \\ pkt_chem@yahoo.co.in (P. K. Tripathy) \\ ${ }^{*}$ Corresponding author \\ To cite this article: \\ Gunanka Hazarika, Pradeep K. Tripathy. Solvent Free Quick Conversion of Mono - And Dicarboxylic Acids into Their Corresponding \\ Anilides with Phenylisothiocyanate. American Journal of Heterocyclic Chemistry. Vol. 5, No. 1, 2019, pp. 4-6. \\ doi: 10.11648/j.ajhc.20190501.12
}

Received: January 25, 2019; Accepted: March 15, 2019; Published: April 8, 2019

\begin{abstract}
The attraction of isothiocyanates as synthons and as cyclizing agents continues due to their diverse reactions and also due to their easy availability. It would not be out of place to mention that, in comparison to isocyanates $(-\mathrm{N}=\mathrm{C}=\mathrm{O})$, their Sulphur analogues, isothiocyanates $(-\mathrm{N}=\mathrm{C}=\mathrm{S})$, are less unpleasant and to some extent less hazardous. The use of isocyanates is drastically limited by the researchers [6] after December 3, 1984 which is the date of Bhopal Disaster held in Union carbide factory, Bhopal, Madhya Pradesh (India) due to the leakage of Methyl isocyanate (MIC) where thousands of people were died due to the toxic effect of MIC (Me-N=C=O). In the present study, a mixture of Phenyl isothiocyanate (2) and monocarboxylic acid (1), in the ratio of 1:1 and Phenyl isothiocyanate (2) and dicarboxylic acid (4) in the ratio of 2:1 are taken for condensation reaction by heating at $160^{\circ}-170^{\circ} \mathrm{C}$ for 15 minutesunder solvent free condition. Pyridine was used as a catalyst/ base in both the cases. The products obtained were monoanilides (3) and dianilides (5) of mono- and dicarboxylic acids respectively which were recrystallized from aqueous ethanol. Dicarboxylic acids gave unexpected results in some of the cases. For example, Phthalic acid produces N-phenylphthalimide irrespective of the molar ratio of the acid and Phenyl isothiocyanate whereas maleic acid produces neither mono- nor dianilides with Phenyl isothiocyanate under the present condition. A proper systematic investigation was carried out towards the condensation of Phenyl isothiocyanate with mono- and dicarboxylic acids.
\end{abstract}

Keywords: Monocarboxylic Acids, Dicarboxylic Acids, Phenyl Isothiocyanate, Pyridineand Solvent Free

\section{Introduction}

There are several methods $[1,2,10]$ available for the conversion of mono- and dicarboxylic acids into their Nsubstituted amides. With a view to achieving a convenient and rapid synthesis of $\mathrm{N}$-phenyl alkanamides, the reactions of several mono- and dicarboxylic acids with phenyl isothiocyanatehave been studied.

The condensation reaction was carried out by heating at $160^{\circ}-170^{\circ} \mathrm{C}$ for 15 minutes, a mixture of Phenyl isothiocyanate (2) and monocarboxylic acid (1), with the ratio of 1:1 and Phenyl isothiocyanate (2) and dicarboxylic acid (4) in the ratio of 2:1. Pyridine was used as a catalyst in both the cases. The products obtained were monoanilides (3) and dianilides (5) which were recrystallized from aqueous ethanol.

\section{Method}

All the prepared compounds are known in the literature [3, $4,7,8,9]$. The purity of the compounds was verified by TLC (silica gel/benzene and ethylacetate with ratio 9:1) and their melting points. Melting point was recorded by metal block melting point apparatus and is uncorrected. The IR spectra of the compounds were recorded on Nicolet is5 Thermo Fisher Scientific, USA.

General Procedure

\subsection{Synthesis of Anilides of Monocarboxylic Acids (3)}

Phenyl isothiocyanate (2) and the monocarboxylic acid (1), 
taken in the ratio of $1: 1$ in the presence of pyridine as a catalyst, were heated at $160^{\circ}-170^{\circ} \mathrm{C}$ for about 15 minutes with constant stirring. The reaction mixture was thoroughly mixed, cooled and the residue repeatedly washed with petroleum ether $\left(40-60^{\circ}\right)$ to remove unreacted phenyl isothiocyanate. The unreacted carboxylic acid was removed by treatment with aqueous sodium bicarbonate followed by washing with distilled water. The respective monoanilide (3) was isolated and the crude product was recrystallized from aqueous ethanol. The relevant data are given in Table-1.

\subsection{Synthesis of Dianilides of Dicarboxylic Acids (5)}

Phenyl isothiocyanate (2) and the dicarboxylic acid (4) taken in the ratio of 2:1 respectively in the presence of pyridine as a catalyst, were heated at $160^{\circ}-170^{\circ} \mathrm{C}$ for about 15 minutes with constant stirring. The reaction mixture was thoroughly mixed, cooled and the residue repeatedly washed with petroleum ether $\left(40-60^{\circ}\right)$ to remove unreacted phenyl isothiocyanate. The mixture was then subsequently treated with benzene to remove anhydride, if any. The unreacted carboxylic acid was removed by treatment with aqueous $\mathrm{NaHCO}_{3}$, followed by washing with distilled water. The respective dianilide (5) was isolated and the crude product was recrystallized from aqueous ethanol. The relevant data are given in Table- 2 .

\section{Results and Discussions}

The condensation of carboxylic acids with isothiocyanates proceeded through addition-elimination reactions to yield amides. Phenyl isothiocyanate (2) gave mono- and dianilides with mono- and dicarboxylic acids respectively. Pyridine as a catalyst/base facilitated the reaction. Apparently, this is owing to the fact that pyridine as a base enhance the concentration of the carboxylate ion which is the reactive species towards phenyl isothiocyanate.

Monoanilides are formed by condensation of monocarboxylic acid with aniline which can not arise by the decomposition of the phenyl isothiocyanate by water as phenyl isothiocyanate does not react with water. To rationalize the monoanilide formation by the interaction monocarboxylic acids with phenyl isothiocyanate in the presence of pyridine at $160^{\circ}-170^{\circ} \mathrm{C}$, the following mechanism is proposed.

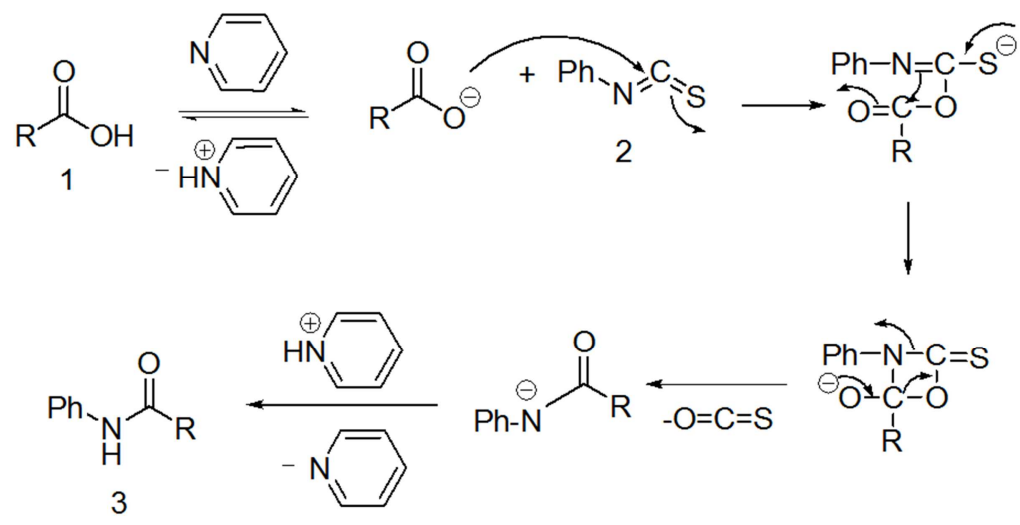

Figure 1. Synthesis of anilides of monocarboxylic acids.

Table 1. Physical data of the anilides of monocarboxylic acids.

\begin{tabular}{lllll}
\hline \multirow{2}{*}{ Compound 3 } & $\mathbf{R}$ & \multirow{2}{*}{ Yield (\%) } & Melting Point $\left({ }^{\circ} \mathbf{C}\right)$ & Reported $^{3,4,5}$ \\
\cline { 4 - 5 } & & 80 & Found & 114 \\
(a) & $\mathrm{CH}_{3}$ & 75 & $110-112$ & 118 \\
(b) & $\mathrm{Ph}^{-\mathrm{CH}_{2}}$ & 68 & $158-116$ & 162 \\
(c) & $\mathrm{Ph}$ & 57 & $121-123$ & 125 \\
(d) & $2-\mathrm{CH}_{3} \cdot \mathrm{C}_{6} \mathrm{H}_{4}$ & 42 & $136-138$ & 135 \\
(e) & $2-\mathrm{OH}_{2} \mathrm{C}_{6} \mathrm{H}_{4}$ & 71 & $212-214$ & 211 \\
(f) & $4-\mathrm{NO}_{2} . \mathrm{C}_{6} \mathrm{H}_{4}$ & 80 & $150-152$ & 153 \\
(g) & $\mathrm{PhCH}=\mathrm{CH}$ & & \\
\hline
\end{tabular}

Table 2. Physical data of the dianilides of dicarboxylic acids.

\begin{tabular}{|c|c|c|c|c|}
\hline \multirow[b]{2}{*}{ Product } & \multirow[b]{2}{*}{$-\left(\mathrm{CH}_{2}\right)_{n^{-}}$} & \multirow{2}{*}{ Yield (\%) } & \multicolumn{2}{|l|}{ Melting Point $\left({ }^{\circ} \mathrm{C}\right)$} \\
\hline & & & Found & Reported $^{3,4}$ \\
\hline 5 (a) & $\mathrm{n}=0$ & 43 & $146-148$ & 148 (monoanilide) \\
\hline 5 (b) & $\mathrm{n}=2$ & 50 & 223-225 (wthoutpy) & $225-227$ \\
\hline $5(\mathrm{c})$ & $\mathrm{n}=4$ & 74 & $238-240$ & 239 \\
\hline $5(d)$ & & 63 & $>300$ & 337 \\
\hline
\end{tabular}

(a)n=0;(b)n =2; (c)n=4;(d) $\left(\mathrm{CH}_{2}\right)=$ 
<smiles>O=C(O)CCCCCC(=O)O</smiles><smiles>C1CCCCC1</smiles><smiles>O=C([O-])CCCCC(=O)[O-]</smiles>

4

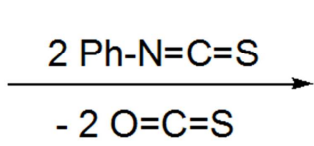

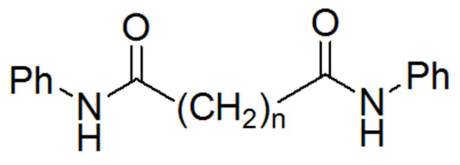

5

Figure 2. Synthesis of dianilides of dicarboxylic acids.

Dicarboxylic acidsafford very interesting results with phenyl isothiocyanate. All the dicarboxylic acids (4) afford the corresponding dianilides irrespective of the molar ratio of the reactants. But phthalic acidforms N-phenylphthalimide (Yield: $60 \%$, m.p. $200-202^{\circ} \mathrm{C}$ ) where phenyl isothiocyanate acts as a cyclising agent. The yields of the dianilides increase when two moles of the phenyl isothiocyanate are used for one mole of dicarboxylic acid. It is observed that the presence and absence of pyridine plays an important role during the preparation of anilides with dicarboxylic acids. The reaction of phenyl isothiocyanate and succinic acid (5b) in $2: 1$ molar ratio without pyridine at $160^{\circ} \mathrm{C}-170^{\circ} \mathrm{C}$ affords the dianilide of succinic acid(Yield; $50 \%$, m.p. $223-225^{\circ} \mathrm{C}$ ) whereas addition of pyridine under similar conditions furnishes a mixture of mono- and dianilides of succinic acid which could not be separated by fractional crystallization. The reaction of phenyl isothiocyanate and oxalic acid dehydrate (5a) in 2:1 molar ratio either in the presence or absence of pyridine affords oxanilic acid (monoanilide of oxalic acid) only (Yield: $50 \%$, m.p. $146-148^{\circ} \mathrm{C}$ ) and this method fails to produce dianilide of oxalic acid. The formation of mono- and dianilides is detected by TLC (Silica gel /Benzene: Ethylacetate =9: 1). However, maleic acid fails to provide expected any type of anilides by using this method. A solid deep brown mass is produced that may be a polymeric substance due to the presence of carbon-carbon double bond in the parent acid which could not be analysed. Adipic acid dianilide (Yield: $74 \%$, m.p. $248-250^{\circ} \mathrm{C}$ ), Terephthalic acid dianilide (Yield: $63 \%$, m.p. $337^{\circ} \mathrm{C}$ ) were obtained conveniently by this method using adipic acid (5c) and terephthalic acid (5d) respectively with phenyl isothiocyanate with the ratio of $1: 2$.

All the compounds, reported $[5,7,8,9]$ were characterized by IR data and by comparison with reported melting point. The characteristic IR for anilides is observed at $\lambda_{\max }(\mathrm{KBr})$ : 3200-3300(NH), 1660-1700(CO, amide) $\mathrm{cm}^{-1}$.

The direct conversion of mono- and dicarboxylic acids into their mono- and dianilides respectively by using phenyl isothiocyanate under solvent free condition seems to be profitable; it overcomes some of the disadvantages of other methods. For example, a large excess of some of the reactants can be avoided and the reaction time is very short. Also, workup for the isolation of anilides is easy. The present method is even successful with phenolic acids like salicylic acids where free $-\mathrm{OH}$ group remains intact in anilide derivative $\left[\lambda_{\max }(\mathrm{KBr}): 3568(\right.$ free $\left.\mathrm{OH})\right]$.

\section{Conclusion}

A straight forward and a fast method for the synthesis of monoanilides and dianilides of mono- and dicarboxylic acids were developed under solvent free conditions. It is noteworthy that the reaction is successful even in the case of phenolic acids, such as salicylic acid, and the product can be separated in a pure state in most of the cases. In view of the simplicity and speed of the reaction, the method can be regarded as potentially important.

\section{References}

[1] Sandler SR and Karo W, "Organic functional group preparations", Ed. Blomquist AT, Academic Press, New York, $1963 ; 269$.

[2] Garcia J and Vilarrasa J, Tetrahedron Lett, 1982; 23: 1127-28.

[3] Ram RN, AshareRam and Mukerjee AK, Chemistry and Industry (London) 1983; 569.

[4] Ashare Ram, Ram RN and Mukerjee AK, Indian J. Chem. 1984; 23B: 759-60.

[5] Vogel AIA 'Textbook of Practical Organic Chemistry', 5 ${ }^{\text {th }}$ Edition, Addison Wesley Longman Limited, UK, 1989.

[6] Mukerjee AK and Ashare Ram, Chemical Reviews 1991; 91 : $1-24$.

[7] 'CRC Handbook of Chemistry and Physics', Edited by Lide David R., $88^{\text {th }}$ Edition (2007-2008).

[8] 'A Handbook of Organic Analysis: Qualitative and Quantitative', Clarke HT, $4^{\text {th }}$ Edition (1927), Published by Wiley International, London.

[9] 'Practical Organic Chemistry', Mann Frederick George and Saunders Bernard Charles, $4^{\text {th }}$ Edition (1960), Published by Longman Inc., New York.

[10] Drobnica L, Kristian P and Augustin J, 'The Chemistry of cyanates and their thio-derivatives' Part 2, Ed Patai S, New York, John Wiley and Sons, 1977;1003. 